\section{Transcutaneous electrical nerve stimulation: current status of evidence}
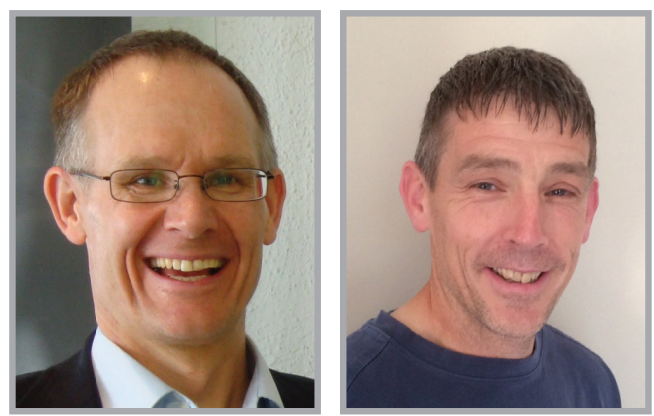

"Practitioners should be mindful that recommendations not to offer transcutaneous electrical nerve stimulation are based on a paucity of evidence on which to make a judgment rather than evidence of inferiority or equivalence to placebo.”

Mark I Johnson ${ }^{*, 1}$ \& Gareth Jones

First draft submitted: 5 July 2016; Accepted for publication: 9 September 2016; Published online: 19 September 2016

In the UK, NICE is in the processes of updating its guidance on the early management of nonspecific low back pain and sciatica. The draft recommendations were released for public consultation on 24 March 2016 and concluded that all forms of exercise including stretching, strengthening, aerobic or yoga should be used in the first instance in conjunction with encouraging people to continue normal activities of daily living [1]. Physical and psychological therapies were also recommended for people who did not improve with previous treatments. The draft guidance states "Do not offer TENS (transcutaneous electrical nerve stimulation) for managing nonspecific low back pain with or without sciatica," based on a paucity of randomized controlled clinical trials (RCTs) on which to judge clinical efficacy. This not only raises a doubt about the clinical efficacy of TENS for nonspecific low back pain but also for the relief of pain for other conditions. Interestingly, in 2016 a systematic review of 13 studies (267 patients) evaluating TENS for chronic low back pain found that TENS provided a significant reduction in pain for 2-24 weeks [2]. The aim of this editorial is to analyze the current status of evidence for TENS for pain relief.

TENS is a technique that involves the delivery of mild pulsed electrical currents across the intact surface of the skin to stimulate low threshold nerves to relieve pain. A variety of healthcare practitioners (e.g., physicians, nurses, midwives and physiotherapists) offer TENS as a standalone treatment or combined with medication for symptomatic relief of inflammatory, neuropathic and musculoskeletal pain. TENS is popular with patients and practitioners because it is inexpensive, available without prescription, easy to selfadminister and safe, with limited potential for toxicity or overdose [3]. One should be vigilant to claims by manufacturers that advanced technology incorporated into device design enables specific interactions with physiological mechanisms because any relationship between specific characteristics of electrical stimulation and physiological mechanisms may not influence

\section{KEYWORDS}

- neuromodulation - pain • TENS

- transcutaneous electrical nerve stimulation

\author{
"Current research evidence \\ suggests that it is reasonable to \\ offer transcutaneous electrical \\ nerve stimulation as an adjunct to \\ core treatment for most painful \\ conditions, especially because it is \\ inexpensive and has a favorable \\ safety profile compared with \\ long-term medication.”
}


"Whether costs associated with transcutaneous electrical nerve stimulation devices and accessories is covered by healthcare providers or patients is a matter for policymakers but practitioners need to be able to advise and educate patients about transcutaneous electrical nerve stimulation because patients may purchase transcutaneous electrical nerve stimulation without prescription." clinical outcomes to any meaningful effect. Psychosocial factors have a strong influence on pain response. Thus, prescriptive, overcomplicated TENS technique should be avoided. Long-term users of TENS report that administering currents to generate a strong nonpainful TENS sensation at the site of pain (conventional TENS) is beneficial.

Uncertainty about the usefulness of TENS has persisted since it was introduced into mainstream medicine in the early 1970s. Since then published research on the mechanisms of action, factors influencing response, clinical efficacy and clinical effectiveness have risen exponentially. Randomized placebo-controlled clinical trials are used to assess clinical efficacy. To answer the question "Do you need to put batteries in the TENS device to get beneficial effects?" investigators could compare real TENS against placebo TENS administered using a sham TENS device that delivers no current. A free-text search in PubMed (1 July 2016) using the term 'transcutaneous electrical nerve stimulation' AND 'pain' found 682 hits for RCT, 195 hits for systematic review and 49 hits for meta-analysis. The majority of systematic reviews on TENS find inconclusive or conflicting evidence for clinical efficacy due to insufficient good quality RCTs. However, subgroup analyses suggest that TENS is superior to placebo when administered using strong nonpainful stimulation within or close to the site of pain (i.e., appropriate TENS technique) [4,5].

The first systematic reviews on TENS were published in 1990s and challenged the belief that TENS was efficacious for acute pain [6,7]. The first meta-analysis on TENS was conducted in 2003 and found that TENS reduced postoperative consumption of analgesic medication to a greater extent than placebo when TENS was administered using appropriate technique [5]. More recent systematic reviews confirm the efficacy of TENS for post-thoracotomy and poststernotomy pain $[8,9]$. The most recent Cochrane review on TENS for acute pain in adults included 19 RCTs (1346 participants) of procedural (cervical laser treatment, venepuncture, screening flexible sigmoidoscopy) and nonprocedural pain (postpartum uterine contractions and rib fractures) [10]. A meta-analysis of six RCTs found that TENS reduced pain intensity by $31.79-17.46 \mathrm{~mm}(95 \% \mathrm{CIs})$ on a $100-\mathrm{mm}$ visual analog scale compared with placebo. The likelihood of achieving $\geq 50 \%$ reduction in pain was 2.42-6.32 in favor of TENS over placebo
(95\% CIs for relative risk, four RCTs). However, there was a high risk of bias due to inadequate sample sizes and unsuccessful blinding of treatment interventions so reviewers concluded that evidence was only tentative. Evidence to support the use of TENS for pain during childbirth is also tentative due to conflicting findings, although women receiving TENS are more 'satisfied' than those receiving placebo [11]. There is insufficient evidence to judge with any certainty the efficacy of TENS for dysmenorrhea, angina pectoris-like chest pain and pain from lacerations, fractures, hematomas, contusions and dental procedures.

The largest meta-analysis on TENS was conducted a decade ago and provides evidence that TENS provides three-times more relief of pain than placebo for chronic musculoskeletal conditions [12]. The analysis included 29 RCTs with 335 participants receiving placebo, 474 participants receiving TENS or percutaneous electrical nerve stimulation and 418 participants crossed over to receive both active placebo electrical nerve stimulation. There were 32 comparisons on TENS. The review methodology was criticized for combining multiple diseases at the expense of homogeneity. To date, there are Cochrane and non-Cochrane systematic reviews on TENS for chronic pain, cancer pain, knee osteoarthritis, rheumatoid arthritis, neck pain, low back pain, chronic and recurrent headache, neuropathic pain, postamputation pain, poststroke pain, diabetic peripheral neuropathy, spinal cord injury and central pain associated with multiple sclerosis. The majority of these reviews conclude that meaningful judgments on the clinical efficacy of TENS are not possible because RCTs were of insufficient quantity or had a high risk of bias associated with methodology. This concern is not unique to TENS as a review of 34 treatments (76 RCTs) for nonspecific chronic low back pain found that study sample sizes were extremely low for most treatments [13].

There are more systematic reviews than RCTs evaluating TENS for some chronic pain conditions. For conditions where sufficient RCTs exist, there is concern about trial fidelity with inadequate TENS technique and dosage common in RCTs [14]. The contradictory nature of evidence provided by TENS research has caused apparent inconsistencies in professional and regulatory body recommendations creating uncertainty for practitioners. For example, it is recommended in NICE guidelines that TENS should be offered for short-term relief of osteoarthritis [15], rheumatoid arthritis [16] and musculoskeletal pain secondary 
to multiple sclerosis [17], but not for nonspecific low back pain [18]. Practitioners should be mindful that recommendations not to offer TENS are based on a paucity of evidence on which to make a judgment rather than evidence of inferiority or equivalence to placebo.

TENS should be considered a complex intervention influenced by a variety of factors, including the choice of TENS settings and pattern of TENS usage. The possible combinations of electrical output characteristics even on the simplest of TENS devices is vast, and the search for 'optimal stimulator settings' has created a body of research literature that is impossible to interpret. This has fueled myths about how best to use TENS in clinical practice. Research from studies using pain-free participants exposed to experimentally induced pain suggest that favorable responses are obtained when the intensity of TENS is strong but not painful and administered at the site of pain (often termed 'conventional TENS'). Thus, practitioners need to educate individuals about safe and appropriate TENS technique, including the need for patients to regularly self-administer TENS. Some patients stop using TENS because they find that the amount of effort needed to administer treatment is disproportionate to the amount of pain relief achieved. Recently, Gladwell et al. [19] conducted semi-structured interviews with nine patients to explore their experiences of using TENS to manage chronic musculoskeletal pain. Challenges to optimize the use of TENS included frequently having to adjust electrode position and TENS settings to maintain pain relief. This research demonstrated the need to incorporate a learning phase to allow patients to optimize TENS usage. TENS success is optimized when new TENS users have realistic expectations from treatment and are provided with strategies to enable them to sustain motivation to self-administer treatment and troubleshoot declining response. A framework to support this approach is available in the book "Transcutaneous Electrical Nerve Stimulation (TENS). Research to Support Clinical Practice” [20].

Trying to interpret research on TENS is at best challenging and at worst futile because synthesizing research findings is hindered by inconsistent terminology, variability in clinical technique and poor study design. Current research evidence suggests that it is reasonable to offer TENS as an adjunct to core treatment for most painful conditions, especially because it is inexpensive and has a favorable safety profile compared with long-term medication. Whether costs associated with TENS devices and accessories is covered by healthcare providers or patients is a matter for policymakers but practitioners need to be able to advise and educate patients about TENS because patients may purchase TENS without prescription. Investigators may consider the use of an enriched enrollment, randomized withdrawal design using a two-stage recruitment process in future RCTs.

\section{Financial \& competing interests disclosure}

MI Johnson receives royalties for his recent book

'Transcutaneous Electrical Nerve Stimulation (TENS).

Research to Support Clinical Practice'. The authors have no other relevant affiliations or financial involvement with any organization or entity with a financial interest in or financial conflict with the subject matter or materials discussed in the manuscript apart from those disclosed.

No writing assistance was utilized in the production of this manuscript.

\section{References}

Papers of special note have been highlighted as: - of interest; $\bullet$ of considerable interest

1 Franklin R. Low back pain and sciatica: management of non-specific low back pain and sciatica. Assessment and non-invasive treatments - Draft for consultation. National Institute for Health and Care Excellence, 1-819 (2016).

www.nice.org.uk

2 Jauregui JJ, Cherian JJ, Gwam CU et al. A meta-analysis of transcutaneous electrical nerve stimulation for chronic low back pain. Surg. Technol. Int. 28, 296-302 (2016).
3 Johnson MI, Bjordal JM. Transcutaneous electrical nerve stimulation for the management of painful conditions: focus on neuropathic pain. Expert Rev. Neurother. 11(5), 735-753 (2011).

- Comprehensive review.

4 Bjordal JM, Klovning A, Ljunggren AE, Slordal L. Short-term efficacy of pharmacotherapeutic interventions in osteoarthritic knee pain: a meta-analysis of randomised placebo-controlled trials. Eur. J. Pain 11(2), 125-138 (2007).

5 Bjordal JM, Johnson MI, Ljunggreen AE. Transcutaneous electrical nerve stimulation (TENS) can reduce postoperative analgesic consumption. A meta-analysis with assessment of optimal treatment parameters for postoperative pain. Eur. J. Pain 7(2), 181-188 (2003).

-• First meta-analysis with a subgroup analysis of optimal delivery of transcutaneous electrical nerve stimulation (TENS).

6 Carroll D, Tramer M, Mcquay H, Nye B, Moore A. Randomization is important in studies with pain outcomes: systematic review of transcutaneous electrical nerve stimulation in acute postoperative pain. Br. J. Anaesth. 77(6), 798-803 (1996).

7 Carroll D, Tramer M, Mcquay H, Nye B, Moore A. Transcutaneous electrical nerve 
stimulation in labour pain: a systematic review. Br. J. Obstet. Gynaecol. 104(2), 169-175 (1997).

8 Freynet A, Falcoz PE. Is transcutaneous electrical nerve stimulation effective in relieving postoperative pain after thoracotomy? Interact. Cardiovasc. Thorac. Surg. 10(2), 283-288 (2010).

9 Sbruzzi G, Silveira SA, Silva DV, Coronel CC, Plentz RD. Transcutaneous electrical nerve stimulation after thoracic surgery: systematic review and meta-analysis of 11 randomized trials. Rev. Bras. Cir. Cardiovasc. 27(1), 75-87 (2012).

10 Johnson MI, Paley CA, Howe TE, Sluka KA. Transcutaneous electrical nerve stimulation for acute pain. Cochrane Database Syst. Rev. (6), CD006142 (2015).

- Most recent Cochrane review on TENS.

11 Dowswell T, Bedwell C, Lavender T, Neilson JP. Transcutaneous electrical nerve stimulation (TENS) for pain relief in labour. Cochrane Database Syst. Rev. (2), CD007214 (2009).

12 Johnson M, Martinson M. Efficacy of electrical nerve stimulation for chronic musculoskeletal pain: a meta-analysis of randomized controlled trials. Pain 130(1-2), 157-165 (2007).
- Largest meta-analysis to date.

13 Machado LA, Kamper SJ, Herbert RD, Maher CG, Mcauley JH. Analgesic effects of treatments for non-specific low back pain: a meta-analysis of placebo-controlled randomized trials. Rheumatology (Oxford) 48(5), 520-527 (2009).

14 Bennett MI, Hughes N, Johnson MI. Methodological quality in randomised controlled trials of transcutaneous electric nerve stimulation for pain: low fidelity may explain negative findings. Pain 152(6), 1226-1232 (2011).

- Reveals methodological quality shortcomings of existing clinical trials on TENS.

15 National Institute for Health and Care Excellence. Osteoarthritis: care and management. NICE guidelines [CG177]. 1-37 (2014). www.nice.org.uk

16 National Institute for Health and Clinical Excellence. NICE clinical guideline 79. Rheumatoid arthritis: the management of rheumatoid arthritis in adults. 1-35 (2009). www.nice.org.uk/guidance/cg79
17 National Institute for Health and Clinical Excellence, Conditions TNCCFC. NICE clinical guideline 8. Multiple Sclerosis. National clinical guideline for diagnosis and management in primary and secondary care (2003).

www.nice.org.uk

18 National Institute for Health and Clinical Excellence. NICE clinical guideline 88. Early management of persistent non-specific low back pain, 1-25 (2009). www.nice.org.uk/guidance/cg88

19 Gladwell PW, Badlan K, Cramp F, Palmer S. Problems, solutions, and strategies reported by users of transcutaneous electrical nerve stimulation for chronic musculoskeletal pain: qualitative exploration using patient interviews. Phys. Ther. 96(7), 1039-1048 (2016).

20 Johnson M. Transcutaneous Electrical Nerve Stimulation (TENS). Research to Support Clinical Practice. Oxford University Press, Oxford, UK (2014).

-• Comprehensive evidence-based book which was highly commended in the Medicine category in the 2015 British Medical Association Book Awards. 